ALPHA Nº 29 Diciembre 2009 (167-184)

ISSN 0716-4254

http://alpha.ulagos.cl

\title{
HACIA LA PREGUNTA POR LA CORPORALIDAD: REFLEXIONES SOBRE EL CUERPO HUMANO EN CUANTO ORGANISMO
}

To the question by the body: Reflections on the human body as organism

Felipe Johnson*

Resumen

Las ciencias naturales y su propagación en las esferas de la vida cotidiana han consolidado una experiencia del cuerpo humano que hoy en día parece ser preponderante, a saber, que éste es un organismo. En su articulación actual, este concepto pareciera ser el fundamento y la directriz indiscutible en gran parte de las consideraciones sobre esta problemática, incluso al interior de ciertas perspectivas filosóficas enraizadas aún en la interpretación cartesiana del cuerpo humano. El presente artículo pretende indagar en aquellos supuestos que configuran esta experiencia, con el fin de contribuir a un planteamiento estricto de la pregunta filosófica por la corporalidad.

Palabras clave: Cuerpo humano, organismo, vida, extensión, función, mecánica, experiencia, Heidegger, Descartes.

\section{Abstract}

The dissemination of the natural science in everyday life have resulted in a particular experience of human body, which now seems to be predominant: the concept of the human body as an organism. The organism concept, in their current articulation, seems to be the basis and guideline in much consideration of this problematic even inside of some philosophical perspective rooted in the Cartesian interpretation of human body. The aim of this article is to investigate the assumptions, which compose this experience in order to contribute with a strict approach to the Philosophical question of corporality.

Key words: Human body, organism, life, extension, function, mechanics, experience, Heidegger, Descartes.

\section{EL CUERPO: BREVE EXAMEN DE SUS MODOS DE ACCESO}

El fenómeno cuerpo humano pareciera nacer y desplegarse al interior de un sinnúmero de prejuicios que han configurado una manera específica de relacionarnos con la corporalidad humana en general. Se habla, por ejemplo, 
de un cuerpo compuesto de órganos (corazón, pulmones, estómago, etc.,) de un cuerpo que fisiológicamente es descriptible en relación a miembros (cabeza, tronco, extremidades) de un cuerpo que ejecuta funciones determinadas (latidos cardíacos, secreción de hormonas, etc.). Se habla, pues, de un cuerpo que no es meramente físico sino, más bien, de un cuerpo orgánico.

Este cuerpo humano, así descrito, pareciera ser aquel ente desde el cual comienza un modo particular de tematización de la corporalidad. Es, por una parte, aquello mentado en la cotidianeidad, por ejemplo, cuando éste ya no nos acompaña en nuestras acciones, cuando estamos enfermos, cuando sentimos dolor, cuando requiere de nuestra atención para ser atendido. Este cuerpo es, por otra parte, aquello mentado cuando una descripción teórica del mismo se desarrolla. Es el caso, por ejemplo, de la anatomía y de su tendencia a descomponerlo hasta en sus mínimos elementos, donde órganos vitales como el corazón o el estómago son descritos en relación a su estructura muscular, o de la química y su intento por desensamblar estos órganos en unidades menores como proteínas, minerales, etc.

Ciertamente, ha sido la biología moderna la que hoy en día ha contribuido a consolidar esta comprensión, siendo así que iniciar una tematización del fenómeno cuerpo humano - y, en nuestro caso, filosóficamenteal margen de la óptica de lo orgánico pareciera ser inevitable. Que esta concepción orgánica haya ganado hoy en día primacía con respecto a una interpretación de orden exclusivamente físico, al interior de la cual la corporalidad es descriptible en cuanto mera masa extensa, es porque la biología pareciera destacar en dicho fenómeno una dimensión aún más decisiva: su vida.

Pues bien, si hemos comenzado por aludir al ente mentado en el “discurso sobre el cuerpo humano" a modo de lo orgánico - y esto, tanto en la cotidianeidad como en esferas teóricas como la biología, la física o la química - ha sido justamente para enfatizar un modo de acceso común a un fenómeno que, en la actualidad, pareciera haberse consolidado como algo obvio. El problema que nos ocupará a continuación no será, sin embargo, el de buscar una interpretación alternativa a esta idea de corporalidad que, por así decirlo, expanda el horizonte de perspectivas de análisis de la misma. Aclarar cuál es dicho modo de acceso significa para nosotros atender a aquella dirección temática que sirve de sentido a priori a una experiencia específica del cuerpo humano, desde la cual, en definitiva, tanto la biología, como la física, como también la química, se presentan como los diferentes modos de estudio de dicho ente. Así, nuestro interés por realizar una tematización filosófica de la corporalidad no radica en dejar de lado este modo de entenderla, sino, más bien, en interrogarla en sus propios presupuestos con el fin de decidir si es que el cuerpo dado en tal experiencia rinde cuenta, en 
Hacia la pregunta por la corporalidad: reflexiones sobre el cuerpo humano

última instancia, de la manera de ser más originaria del fenómeno cuerpo humano. ${ }^{1}$

\section{HACIA LA PREGUNTA POR LA CORPORALIDAD: EXAMEN DEL CUERPO HUMANO COMO ORGANISMO Y SUS PRESUPUESTOS METÓDICOS}

La discusión acerca del cuerpo humano en tanto organismo se torna relevante para esta investigación en distintos niveles de nuestras reflexiones. Ella es significativa en cuanto a la elucidación del modo concreto como es entendido todo ser viviente, incluyendo al ser humano. El concepto "organismo" nos enseñará, en este contexto, qué es aquello que actualmente implica hablar de un cuerpo vivo. Este nivel es, sin embargo, introductorio y sólo servirá de vía al problema auténtico que implica el intento filosófico de delimitar el fenómeno en cuestión, a saber, mostrar que el concepto "organismo" no nace de la experiencia concreta, sino, más bien, que determina la misma, de tal manera que —en virtud a su articulación - un tipo específico de ente podrá posteriormente ser observado empíricamente. De esta manera entenderemos que este concepto implica fundamentalmente una determinación a priori de todo tipo de tematización teórica de la corporalidad humana. Comprender la naturaleza a priori del concepto “organismo” podrá guiar nuestra investigación, finalmente, a una toma de posición en relación a aquellos elementos fundamentales que caracterizan un modo de acceso preponderante a la corporalidad humana.

Aclarado el puesto metódico que posee el concepto "organismo" en este artículo, atenderemos al primer nivel arriba mencionado, a saber, qué es lo que está implicado en dicho concepto. Como es sabido, lo orgánico, en su sentido biológico moderno, es aquello donde se presenta de modo inherente la vida. A diferencia del cuerpo inerte que exhibe, por ejemplo, una piedra, el cuerpo de un ser orgánico presenta un número determinable de características que se insertan en el horizonte de lo vivo. En otras palabras, existe una relación vinculante entre los conceptos “vida” y “organismo”, que aquí deberá ser expuesta si queremos delimitar qué es lo mentado en el discurso acerca de un ente orgánico. Así es como el concepto "vida” gana en esta discusión una evidente primacía, ya que aquello que comprendamos por "vida” será justamente el criterio último para decidir qué es o no es un organismo. La pregunta que deberá ser resuelta a continuación será la siguiente: ¿Cómo se

\footnotetext{
${ }^{1}$ Es importante destacar que el uso del término "fenómeno cuerpo humano" en esta fase de la discusión guarda un carácter estrictamente formal y, por ende, aún desligado de cualquier concreción. La intención, en esta parte introductoria, es sólo atender a que el fenómeno cuerpo humano puede ser otro y que no es necesariamente vinculante con el cuerpo orgánico descrito en el contexto de la biología o de las ciencias de la naturaleza en general.
} 


\section{Felipe Johnson}

manifiesta la vida en un organismo? Es decir ¿Qué es aquello observable en el ente que, en última instancia, pareciera indicar que éste está vivo? Preguntamos, entonces: ¿Qué es lo vivo en un organismo?

No estaríamos lejos de la respuesta si atendemos al hecho de que todo ser vivo manifiesta una organización. Podría decirse, en primera instancia, que un organismo es una unidad organizada. No obstante, la unidad mentada —en este caso- no permanece en el nivel de la constitución física del ser vivo. Que un animal posea cabeza, extremidades, o que posea corazón o pulmones, no implica necesariamente que esté vivo. Pero sí es importante destacar que la vida en él parece relacionarse con las partes, pues son ellas las que acusan su aludida organización. En este sentido podemos afirmar: corazón, pulmones, cerebro, no son meras unidades físicas, sino aún más, son en sí mismas unidades que ejecutan una función. Los ojos sirven para ver; las manos, para asir o para tocar algo; los oídos, para escuchar. Cada parte del organismo, en cuanto componente de una unidad funcional, ejecuta, en definitiva, un trabajo propio. En este contexto podríamos retrotraer el concepto griego organon a la idea de la producción de una obra, ${ }^{2}$ destacando así en el vocablo griego la presencia de un ergon. ${ }^{3}$ Lo que aquí apreciamos es el realce del modo de ser de todo órgano en su sentido moderno: cada órgano se constituye como tal si concibe la obra que de él es requerida. La producción se nos presenta como aquella manera de ser del ente órgano, es decir, ésta caracteriza aquel modo auténtico como el "ser órgano" se despliega. Los ojos conciben un lugar dentro del cual el movimiento es viable, detectan los obstáculos posibles que deberán ser sorteados mientras hay traslado. El órgano no produce algo para sí mismo, sino más bien en pro del ser vivo, de la unidad, de tal manera que si tuviéramos que precisar la mentada relación de la parte en pos de la unidad deberíamos decir, según lo planteado, que el

\footnotetext{
${ }^{2}$ La referencia a la raíz etimológica del vocablo griego organon al modo de ergon y el énfasis de su significado en el sentido de producción puede ser hallada en Martin Heidegger. Die Grund. der Metaph (2004:312).

${ }^{3}$ Es apropiado, sin embargo, destacar que el recurso a los términos griegos en Heidegger, para delimitar ciertos fenómenos relevantes, no atiende a una filologización del quehacer filosófico donde la palabra ganaría primacía por sobre los fenómenos. Al contrario, si el hablar (sprechen) o decir (sagen) para Heidegger, entendido como mostrar (zeigen) implica, en última instancia, dejar aparecer al ente en cuanto lo que él es, entonces, pareciera que la articulación fáctica del vocablo y, en este contexto, de la palabra griega, se consolida desde un momento de verdad, desde el aparecer de la cosa misma. Si el término organon en este sentido es explicitado en su relación con ergon, dicha labor pareciera, más bien, atender a la búsqueda del suelo experiencial inmediato, es decir, de aquel campo fenoménico auténtico griego, en virtud del cual el vocablo organon habría nacido.
} 
órgano, en cuanto parte, es lo útil para el todo. Al ser del órgano, en cuanto producción de una obra, le será, entonces, inherente el carácter de la utilidad. ${ }^{4}$

Desde esta perspectiva, el órgano, en su articulación moderna, podría ser entendido como una herramienta que presta un servicio al todo, de manera que serían las funciones de las partes aquello que ahora se nos muestra como lo primariamente organizado. De esta manera, observaremos que el concepto "organismo" pareciera resistirse a ser definido como una unidad originaria, como en un comienzo nos parecía oportuno hacerlo. En efecto, en cuanto organización de funciones entre las partes, el organismo muestra ser, antes que nada, un compuesto, una suma de unidades menores, cuyo vínculo les viene dado tardíamente, a saber, mediante el concepto de función. El estómago y los intestinos procesan el alimento de tal manera que éste se descompone en aquello que es beneficioso o prescindible para el ser vivo. Los pulmones filtran el aire para obtener el oxígeno que el mismo requiere. En su conjunto, todas las funciones apuntan a conservar la unidad, es decir, a procurar que dicha unidad siga siendo tal. Pues bien, si tuviéramos ahora que referirnos a dichas funciones podríamos mencionar, por ejemplo, algunas tales como alimentación, reproducción, desarrollo, transmisión hereditaria, crecimiento, estimulación, regulación, movimiento o interacción energética, pues es así como en la actualidad la funcionalidad organizada de las partes pareciera, precisamente, acusar la vida en cada organismo. ${ }^{5}$

Entonces, si nuestra pregunta inicial apuntaba a hacer explícito qué es lo vivo en todo organismo, ahora podemos apreciar que vida y función -en el contexto moderno de la biología - se copertenecen y que dicha copertenencia se traduce en que lo vivo tiene su propio lugar en un ensamblaje de componentes, a saber, al modo de una ejecución conjunta de funciones que conservan un todo. ${ }^{6}$ Es Heidegger, por ejemplo, quien delimita el concepto de vida que actualmente prepondera como Gefüge von Abläufen (2004:317) donde nuevamente se enfatiza que los procesos o funciones (Abläufe) no son un fin en sí mismos sino que adquieren su propio rol al interior de un entramado (Gefüge) del cual cada uno es un componente.

\footnotetext{
${ }^{4}$ Seguimos acá la determinación hecha por Heidegger en su lección Die Grundbegriffe der Metaphysik. (Cfr., sobre todo, las páginas 320 y 321).

${ }^{5}$ Así es como pensadores que han concentrado sus esfuerzos en delimitar formalmente el campo de estudio de la biología — pensemos, por ejemplo, en Helmuth Plessner — establecen los criterios que acusarían la vida para la tradición biológica (1975:112).

${ }^{6}$ Plessner destaca la comprensión tradicional de vida a la manera como la articula Bichat en sus Recherches physiologiques sur la vie et la mort: "la vida es un ensamble de funciones que se resisten a la muerte” (1832:2). (Esta traducción y las siguientes son mía). En efecto, para el fisiólogo francés, la vida orgánica no consiste más que en un ensamblaje de reacciones que luchan contra la constante destrucción a la cual el organismo, inserto en un entorno, está expuesto.
} 


\section{Felipe Johnson}

Decisivo será observar, en este punto, que el concepto "organismo" en su copertenencia con el concepto "vida" remite inmediatamente a la idea de una máquina, pues la precomprensión de vida como función no podría ser entendida sino como el resultado de la interacción funcional entre partes. Dicha idea de vida parece, en conclusión, servir de criterio último para decidir cuándo podemos hablar de un ser vivo y cuándo no y, esto, ciertamente, desde una perspectiva temática específica: lo mecánico.

En definitiva, si el primer nivel de nuestra discusión debía rendir cuenta de aquello implicado en el discurso actual sobre un cuerpo vivo, pareciera ahora ser adecuado atender a la mecánica, pues ciertamente los conceptos utilizados a lo largo de la presente discusión, como "ejecución”, "interacción”, “ensamblaje”, "función”, "trabajo conjunto de las partes" no han emergido sino mediante esta área de la Física moderna. ${ }^{7}$ De hecho, si atendemos al origen histórico de la concepción de vida hasta ahora descrita, encontraremos justamente ahí la necesidad explícita de la intervención de dicha disciplina en el estudio de los animales y, por ende, también del hombre. Nos referimos ciertamente a Descartes y en particular a $\mathrm{La}$ Description du corps humain (1664) donde afirma

Pero, porque hemos experimentado, desde nuestra infancia, que muchos de sus movimientos obedecerían a la voluntad, que es una de las facultades del alma, aquello nos ha dispuesto a creer que el alma es el principio de todos. A lo cual también ha contribuido mucho la ignorancia de la anatomía y de la mecánica (1996:224).

Es importante observar, a partir de la cita, que el hecho de que Descartes resalte la ignorancia de la mecánica no debe ser interpretado como un intento de desacreditar su rol en el estudio del cuerpo, esto es, en la anatomía. Por el contrario, su afirmación pareciera apuntar, más bien, a la exigencia de perfeccionarla, de manera que sea superado un estadio de conocimiento inicial de la corporalidad aún enraizado en supuestos inaceptables. Según la cita, sería esta relación dudosa del cuerpo con el alma la que debería superarse para poder así consolidar, definitivamente, un estudio estricto del cuerpo humano. No obstante, si el papel de Descartes se torna significativo en la presente discusión precisaremos, más adelante, en qué

\footnotetext{
${ }^{7}$ Heidegger enfatiza que la comprensión que predomina en la psicología sobre la voluntad entendida como Trieb (impulsión, inclinación) atiende, ya, al ente en un sentido mecánico y que, por lo mismo, es inconciliable con la manera de ser auténtica del hombre, el Dasein: "Los intentos explicativos de fenómenos humanos a partir de los impulsos poseen el carácter metódico de una ciencia cuyo campo de estudio no es en absoluto el hombre, sino la mecánica” (1994:217).
} 
radica específicamente su relevancia. Es así como el análisis del concepto “organismo" nos lleva al segundo nivel de nuestra investigación, a saber, profundizar en la manera como la experiencia del cuerpo humano se ha visto determinada mediante el concepto de organismo.

Tal como hemos descrito aquello que implica hablar sobre un organismo pareciera ser, en efecto, el modo como cada ente orgánico aparece en la experiencia concreta actual. La experiencia cotidiana nos enseña que nuestro cuerpo posee órganos que interactúan entre sí, nos muestra que éste es divisible fisiológicamente. Independientemente de la convicción particular de que nuestra vida no consista sólo en el funcionamiento correcto de este ensamblaje, ${ }^{8}$ la experiencia nos muestra que el despliegue de nuestra existencia concreta, que la realización de nuestros planes vitales, dependen del buen estado de nuestro cuerpo. ${ }^{9}$ Precisamente, esta comprensión de cuerpo es la que parece ser incuestionable y a partir de la cual todo tipo de consideración de la corporalidad debiera partir. Este modo de experiencia del cuerpo es el que se ha consolidado con un carácter de obviedad.

De esta manera llegamos al centro de la problemática que implica la consideración filosófica del cuerpo humano. Interrogar su obviedad constituye, a nuestro parecer, uno de los pasos metódicos necesarios para la preparación de su pregunta. No solamente porque el cuerpo, en esta obviedad, ya no requiere ser nuevamente interrogado, sino, más bien, debido a un problema aún más de fondo: lo obvio implica una articulación concreta del ente que se ha vuelto absoluta. Esta obviedad remite, así, a una costumbre de conocer al cuerpo que (al momento de volverse tal, es decir, de consolidarse) gobierna todo escuchar, esto es, todo "conocer del fenómeno". ${ }^{10}$ Nos hallamos,

\footnotetext{
${ }^{8}$ En una breve conferencia titulada Die Idee des Arztes, K. Jaspers expone la modificación que ha tenido la relación entre médico y paciente a partir de la inserción del concepto "humanidad" en la medicina. En este sentido, la vida pareciera desplegarse en un ámbito que se mantiene al margen de toda concepción física. Sin embargo, la salud corporal es decisiva en este punto como aquello que posibilita la plenitud de dicho despliegue. Cfr. Karl Jaspers. Die Idee des Arztes y también Arzt und Patient, ambos publicados en un tomo recopilatorio titulado Philosophie und Welt: Rede und Aufsätze (1958).

${ }^{9}$ En este contexto hablamos de buen estado del cuerpo, a saber, como aquella máquina que funciona correctamente, porque el concepto de salud moderno pareciera estar enraizado en esta precomprensión funcionalista de la vida. Así, es posible hallar en Bichat la distinción entre dos tipos de vida: la primera sería la vida orgánica que remite a movimientos internos al cuerpo, producidos por el funcionamiento y la interacción de los órganos; la segunda sería la vida animal que daría cuenta del organismo en su totalidad y de su interacción con su propio medio. Cfr. Bichat (1832:8).

${ }^{10}$ Consideramos esta advertencia como uno de los momentos fundamentales del ejercicio filosófico, en tanto sugiere - aunque ciertamente de manera aún ambigua - que la tendencia de este modo de conocer al ente implica la ruptura con todo ethos. En este punto estamos atendiendo ciertamente a Aristóteles, quien afirma en Metafísica: "Los actos de escuchar van de la
} 


\section{Felipe Johnson}

entonces, ante un modo imperante de relación con el ente, ante un modo dominante de acceder a él. Pues bien, si hemos descrito la comprensión del cuerpo humano al modo de un organismo, y al principio ya decíamos que ella se ha desplegado mediante un sinnúmero de prejuicios, ha sido, pues, para hacer referencia a aquello que ahora entenderemos como aquel modo de acceso a priori que, en última instancia, ha hecho que el cuerpo humano haya sido entendido principalmente del modo como lo hemos expuesto. A partir de lo anterior podemos ahora afirmar que la idea de cuerpo en cuanto ensamblaje de funciones delata, por un lado, un modo específico de la presentación del ente, tal como él se aparece en cada experiencia. Además, por otro lado, delata un modo específico de experiencia, mediante la cual este aparecer se despliega. La idea de organismo - y todos los elementos que pertenecen a su horizonte conceptual - es desde esta perspectiva, aquel sentido específico que caracteriza una relación previa con los seres vivos; relación que, por lo demás, ella misma, se efectúa concretamente en un conocer de los mismos a la manera de máquinas.

Sin embargo, en el marco de las consideraciones anteriores, podemos apreciar que atender a la idea de organismo como máquina no satisface aún el sentido de interrogar por los presupuestos de la experiencia moderna del ser vivo. Todavía queda pendiente la tarea de delimitar cómo es que este aparecer se despliega. La pregunta dirige nuestra atención, ahora, al modo característico de aquella experiencia específica mediante la cual este aparecer se realiza, es decir, al modo específico de tener al ente —que en la experiencia cotidiana se denomina "organismo" - para llegar a tomar conciencia de su propio modo de ser.

Si volvemos nuevamente a la noción de organismo como "ensamblaje de funciones” y recordamos que cada función es ejecutada por los órganos, podremos observar que en dicha noción de cuerpo la parte gana primacía por sobre el todo. De hecho, esta primacía no se aprecia sólo en la división del organismo en órganos, sino también en su división fisiológica. La cabeza, las manos, las piernas resultan ser también divisiones que aprehenden al cuerpo humano de manera más detallada. Por esta vía pareciera, incluso, ser factible afirmar que sin manos, sin piernas o sin cabeza es imposible concebir el cuerpo humano. La pregunta que surge inmediatamente a partir de lo expuesto es ¿Cómo ha sido posible que el cuerpo humano no se entienda como una unidad originaria, sino, más bien, como el resultado tardío de una suma de partes? ¿Cuál es ese modo de acceso al ente que privilegia el rol de las partes ante el todo?

mano según la costumbre, pues estimamos hablar del modo como estamos acostumbrados”. (994b:31-32). 
Tomando en cuenta que cada función es ejecutada por un órgano, es plausible afirmar que ellas requieren, para su ejecución, de un sustento físico. No habrá vista, no habrá oír, sin la existencia previa de un agente físico que ejecute la acción. De ahí que sean los órganos los que adquieren relevancia en las consideraciones biológicas del cuerpo. Ellos son los responsables de la ejecución de las múltiples funciones. En esta discusión sobre el organismo, el concepto que parece ganar primacía última es, precisamente, el de "materia física” y esto en el sentido específico de ser soporte para la ejecución de cada función. El cuerpo humano, en cuanto físico, no parece ser otra cosa sino aquello que materialmente deberá subyacer a toda acción. El cuerpo se nos muestra, a partir de lo anterior, como un ente provisto de alto, ancho y profundidad; un ente que ocupa un punto determinado en el espacio y el tiempo (su entorno) de manera que, atendiendo a su calidad de viviente, es capaz ahora de ejecutar funciones para permanecer presente en ese espacio y tiempo que él rellena. ${ }^{11}$ En otras palabras, el concepto de función, que anteriormente se nos aparecía como principal en la definición de organismo, no parece sino estar enraizado en la consideración física del cuerpo humano. La manera de ser auténtica del mismo, en cuanto organismo, no sería más que la manera de ser de los entes físicos, al cual, sin embargo, le viene dada la funcionalidad para distinguirlo de los seres inertes.

La determinación del cuerpo humano como algo físico pareciera ser indudable. De hecho, la existencia de todo ente es considerada mediante el modo de ser de lo físico y, en este sentido, el todo en el cual el cuerpo se encuentra - la naturaleza - es, antes que nada, algo físico. Antes que un organismo pueda interactuar con su medio, antes de que el animal pueda desplegar su vida en su entorno, le es requerido poseer los atributos de lo físico. Desde esta comprensión, la relación del hombre con su entorno y con los demás hombres se funda en el hecho de que su cuerpo cumpla la función de ser su sustento material. En este contexto, la primacía del cuerpo - como aquella dimensión del ser humano en virtud de la cual éste puede concretar sus planes vitales - pareciera, así, explicarse mediante la primacía de lo físico en el mundo natural, pues, no sería posible hablar de ningún tipo de interacción con otros entes sin que ellos estuviesen efectivamente presentes en un lugar determinado; no podríamos afirmar que en cada animal hay una vida emocional, si lo físico no sirviera a ella de sostén. Incluso, todo tipo de reacción emotiva, todo tipo de padecer es, en cuanto resultado de una

\footnotetext{
${ }^{11}$ Utilizamos el verbo "rellenar" siguiendo a Husserl, para quien la cosa extensa se relaciona con el tiempo y el espacio al modo de un instalarse que ocupa un lugar al interior del continuo espacio-temporal. Husserl ocupa precisamente el verbo "erfüllen" (rellenar) para caracterizar el modo como el ente natural se posiciona en el espacio y en el tiempo (1952:29-32).
} 
interacción, ejecutado por un ente primariamente físico. La vida anímica del ser humano, bajo la concepción del ente natural, no es más que el resultado de la suma de interacciones vividas por un hombre concreto afectado por un mundo concreto. Sin ir más lejos, la vida anímica —en sí misma- pareciera ser entendida primariamente como encapsulada dentro de los límites del cuerpo físico y el hombre, como todo ente natural, se torna, bajo esta comprensión, en su portador. En definitiva, el hombre del mundo natural no es más que una unidad fisio-psíquica, término por medio del cual se realza el carácter de físicamente fundado de todo aquello que no exhibe el carácter de lo "concreto" al interior del mundo natural. ${ }^{12}$

Ahora bien, si nuestra pregunta apuntaba a aclarar el modo de ser del cuerpo humano en su articulación moderna, no podemos sino buscar su respuesta en el modo de ser de lo físico, es decir, en lo que se nos muestra primariamente como algo extenso. La definición preponderante de cuerpo la encontramos nuevamente en Descartes

Por el cuerpo yo entiendo todo aquello que pueda estar delimitado por alguna figura; que pueda estar incluido en algún lugar, y ocupar un espacio de tal forma que todo otro cuerpo sea excluido; que puede ser sentido, o bien por el tacto, o por la vista, o por la audición, o por el gusto, o por el olfato; que puede ser movido de muchas maneras, no por él mismo, mas por algo extraño por el cual éste es tocado y del cual él recibe la impresión (1996:20) (Trad. mía).

Esta cita es significativa para nuestra discusión si atendemos a la nivelación de caracteres corporales que ahí es destacada. En primer lugar, se realzan atributos que pertenecen al cuerpo en sí mismo. Estos son, justamente, los que son aludidos por el concepto "figura", entendida como los límites de la extensión de la res corpórea. Es interesante apreciar que la presencia de los atributos posteriormente enumerados en el fragmento depende de dichos límites. Obtenemos, pues, que el hecho de ocupar un espacio excluye la presencia de otros cuerpos. Podríamos decir que la extensión es acá entendida como todo aquello que es el ente, siendo así que la presencia de aquello que, por el contrario, no lo es deberá situarse fuera de los límites de su extensión, es decir, de lo que él espacialmente abarca. Como podemos ver, el carácter espacial de la res corpórea viene requerido por el largo, el ancho y la profundidad, caracteres que Descartes determinará como los esenciales de

${ }^{12}$ Con la denominación fisio-psíquico, como carácter del ente animal en la naturaleza, atendemos al término alemán "physio-psychisch", utilizado por Husserl, porque éste, en contraposición con el de psicofísico, "es más adecuado para aludir al carácter fundador de lo físico" (1952:183). 
todo cuerpo físico (1996:50) y que, en este contexto, confirman su primacía como momentos constitutivos de la extensión. El carácter espacial aparece, en este sentido, como un atributo que no es inherente a la cosa misma. Éste corresponde, más bien, a un tipo de atributo que pertenece al ente tanto interior como exteriormente, es decir, como el resultado de su estructura interna en vistas a lo que ella misma no es.

En un segundo nivel aparece aquello que se da en virtud de la afección sensible. Importante es ver que estos atributos no pertenecen estrictamente al cuerpo, sino que, más bien, corresponden a las afecciones que se producen en quienes interactúan con él. Este tipo de atributos es, según lo discutido, de orden estrictamente exterior a la constitución propia del cuerpo. Sin embargo, cualquier dato sensible que sea apreciable en éste - y esto es lo relevanterequerirá de la extensión y de la ubicación espacial del cuerpo percibido, pues sin ellos es imposible hablar de interacción que, a nuestro parecer, es precisamente lo que está en juego en los términos tocar, ver, oír, degustar y oler.

En tercer lugar, encontramos en la definición cartesiana de cuerpo una remisión al movimiento, atributo que, por lo demás, presupone todos los anteriores. Como es posible apreciar en lo ya citado, el movimiento es un atributo adquirido mediante la interacción con otros cuerpos, de los cuales (como Descartes expresa) la impresión viene dada. En otras palabras, el movimiento es una disposición asumida por un cuerpo que, sin embargo, nada dice de los caracteres primarios como su largo, ancho y profundidad, sino que, más bien, los requiere para existir. El movimiento de todo cuerpo, podríamos decir ahora, apunta a una condición contingente del mismo y no a una condición esencial.

Lo decisivo del modo como Descartes despliega su definición de cuerpo radica en atender a la relación que existe entre los niveles de atributos corporales que han sido enumerados. Mediante la descripción anterior podemos apreciar que hay una dependencia entre ellos. Sin embargo, si se quisiera delimitar con mayor precisión de qué modo dicha dependencia es entendida, deberíamos observar que los atributos tardíos como lo espacial, lo sensible y el movimiento se apoyan los unos sobre los otros, resultando que la extensión, es decir, el largo, el ancho y la profundidad —o lo que acá aparece bajo el término "figura" - se nos muestra como el último sustento sobre el cual los demás atributos, al modo de capas constitutivas, se van sobreponiendo. Es así como, mediante Descartes podremos obtener algunas observaciones relevantes en vistas a la manera actual de comprender el cuerpo humano.

En primer lugar, habrá que destacar que la relación de dependencia de los atributos caracterizada al modo de apoyo sobre un sustento último contribuye a delimitar el modo de ser específico del cuerpo vivo, pues a este 


\section{Felipe Johnson}

modo de ser le pertenece estrictamente la relación del apoyo, a saber, al estar presente en un espacio determinado. ${ }^{13}$ El cuerpo vivo, y esto aunque se le predique el carácter de vida, no sería más que algo extenso, cuyo rol en la constitución del ente radica precisamente en subyacer para que los demás atributos puedan hacerse presente. Esta relación de dependencia entre atributos se vuelve decisiva para la comprensión del cuerpo como organismo, pues se constituye en una advertencia acerca de los presupuestos que inevitablemente se asumen cuando el cuerpo vivo es entendido como función. Desde esta perspectiva se deberá entender que, aunque el cuerpo no sea más problematizado como extensión (y esto para permanecer en su dimensión funcional) subyacerá necesariamente a dicha tematización su carácter esencialmente extenso como condición de posibilidad de este tipo de interpretación. En otras palabras, el área que éste abarca en su despliegue geométrico es aquello de lo que depende toda función, aquello que en definitiva gana primacía, aunque sea implícitamente, en su constitución y, por ende, en su tratamiento temático.

En este contexto podemos afirmar que el ser de la corporalidad sufre aquí una reducción extrema, impidiendo que la pregunta por su propia esencia pueda seguir siendo desarrollada. Todo lo que es el cuerpo es todo aquello que él abarca geométricamente. Todo lo que él muestra en relación a su constitución esencial (necesaria) es largo, ancho y profundidad. La posibilidad de una ontología del cuerpo humano parece verse, de esta manera, presa en las nociones meramente espaciales del ente sin que pueda dar cuenta, en última instancia, sobre algo más que sobre una mera cosa. A partir de la articulación de la idea de cuerpo en Descartes, el fenómeno cuerpo humano se cosifica, reduciendo su ser más propio a las dimensiones de la extensión que, en definitiva, lo vuelven indistinto a todo otro objeto natural. A partir de la filosofía cartesiana, la posibilidad de una ontología del cuerpo humano —en el sentido de mostrar lo auténtico, lo distintivo del fenómeno- - no puede sino desplegarse como una ontología de la extensión, viéndose así obligada a asumir que el cuerpo humano, en su esencia, no guarda ninguna distinción fundamental con respecto a otros entes naturales, pues todos ellos exhiben en primera instancia la manera de ser de lo físico.

Pues bien, la primacía de lo físico como carácter esencial de la corporalidad parece entenderse a partir de lo anterior. Que el ser humano pueda ser entendido, esencialmente, como un ente compuesto por un cuerpo físico parece implicar necesariamente que a éste le pertenece la posibilidad de instalarse efectivamente en un espacio geométrico. Lo físico significa la posibilidad efectiva de estar presente en un espacio homogéneo, al interior del

${ }^{13}$ Utilizamos acá una traducción para aquel modo de ser que pertenece al ente intramundano y que por Heidegger fue denominado Vorhandenheit (2001:183). 
cual una relación con otros entes recién podrá acaecer. Dicha relación, la interacción entre cuerpos, no es, entonces, el fenómeno principal en la caracterización del cuerpo como res extensa sino, más bien, el hecho de que los entes que interactúan entre sí poseen, en sí mismos, primariamente el carácter de este "instalarse concretamente en el espacio natural". La interacción es pues, como ya veíamos en Descartes, un atributo meramente secundario que, en cuanto requerido por un sustento material, nada dice sobre la esencia de la corporalidad humana. En definitiva, lo físico al interior del mundo natural es lo necesario para toda forma de movimiento del ser vivo y, por lo mismo, para la realización de cada una de sus funciones y de su vida en general. Así, podremos decir que el término "sustento material" no está sino enraizado en la primacía de lo físico como la posibilidad de todo objeto natural de estar presente en un espacio determinado. De esta manera podemos entender que la relevancia que gana el cuerpo en toda discusión en la que se realce su rol de medio necesario para la realización de la vida humana, no vendría dada sino de la comprensión cartesiana de éste como el soporte material último para todo tipo de interacción al interior de un espacio extenso. ${ }^{14}$

Mediante la primacía de lo físico en la consideración natural del cuerpo humano estamos en condiciones de comprender la relación que existe entre el cuerpo y la vida. No podríamos decir sino que esta relación es externa. La vida, entendida como función, es sólo un atributo posterior a la constitución esencial del cuerpo. Bajo la óptica de la función, ella no podría ser abordada más que mediante la cuantificación de movimientos locales. En efecto, la búsqueda de lo eminentemente humano al interior del pensamiento cartesiano $\mathrm{y}$, por ende, de su manera de vivir auténtica se enmarca en sus consideraciones sobre el cogito. El pensar es, pues, aquel modo de ser que caracteriza

\footnotetext{
${ }^{14}$ La idea anteriormente expuesta es, pues, el punto de partida desde el cual Heidegger puede criticar la afirmación de Nietzsche de que el cuerpo sería aquello que "metódicamente debe ser antepuesto", diciendo que en Nietzsche subyace el "cartesianismo más radical y puro que jamás se pueda llegar a pensar". La mentada cita de Nietzsche dice: "El fenómeno del cuerpo es el fenómeno más rico, más claro y tangible: metódicamente ha de ser antepuesto, sin que nada se reproche acerca de su significado último” (1999:205). La crítica de Heidegger, por su parte, dice: "La doctrina de Nietzsche de la primacía metódica del cuerpo vivo (Leib) es el cartesianismo más radical y puro que jamás se pueda llegar a pensar” (1986:247) (Trad. mía). Hay que decir, sin embargo, que la noción de cuerpo en Nietzsche será de suma importancia para las posteriores consideraciones de Heidegger sobre la corporalidad, como se podrá ver en sus seminarios de 1939: Nietzsches Lehre vom Willen zur Macht als Erkenntnis, donde, efectivamente, destaca la problemática del cuerpo vivo (Leib) a partir del pensamiento de Nietzsche, no obstante ahora desplegándola al interior de su modo de ser apropiado, del Dasein y, por ende, desvinculándola de la manera de ser de la res extensa que es, justamente, lo que en la mencionada crítica de Heidegger a Nietzsche y, por cierto, en nuestra actual discusión está en juego.
} 


\section{Felipe Johnson}

esencialmente la naturaleza humana mientras que todo aquello que no es humano, es decir, todo aquello que no exhibe el modo de ser de la res cogitans, no es más que res extensa, como es el caso del propio cuerpo. En definitiva, si debiésemos aclarar los movimientos perceptibles en dicho cuerpo, esto debería, entonces, ser realizado mediante leyes acordes a su propia naturaleza, pues éste, y considerando que la res extensa es esencialmente independiente de la res cogitans, no se regiría bajo la misma legalidad que la del pensar. ${ }^{15}$

Por esta razón, cualquier consideración filosófica que realce la función - y esto, en el sentido de la interacción con otros entes y con el medio; o en el sentido de enfatizar su preeminencia para establecerla como un momento ontológico del cuerpo humano mismo - no hace más que privarse de la posibilidad de interrogar cuál es ese modo de ser específico desde el cual ella podrá ser legítimamente tematizable. Hemos dicho que tal modo de ser es aquel del objeto de estudio de la mecánica, ahora podemos afirmar que dicho objeto de estudio no es otra cosa sino lo físico tomado a partir de su interacción causal y, por cierto, inerte. Desde esta perspectiva, hablar sobre un cuerpo vivo no parece sino significar haber establecido una diferencia cuantitativa con el cuerpo meramente extenso, pues el cuerpo humano se nos muestra, acá, como un ente que posee un atributo añadido, siendo esencialmente igual a todo otro ente físico. Los límites de la vida se nos muestran, en este contexto, determinados por los límites de lo físico, en el sentido de que aquellos se fundan en estos, pero en cuanto función van más allá, se dirigen al exterior de los mismos. ${ }^{16}$ La consideración del ser del cuerpo, en cuanto extensión, relega así lo vivo en él a una dimensión carente de todo tipo de pertenencia interna con el fenómeno en cuestión, con lo cual la relación entre cuerpo y vida no puede ser explicada sino como un mero y, por cierto, oscuro sustentarse.

\footnotetext{
${ }^{15}$ La vinculación exclusiva de la res cogitans al fenómeno de lo humano se vislumbra en la segunda meditación de Meditaciones Metafísicas. En un intento de evadir la tarea de obtener la esencia del hombre a partir de la definición animal rationale, pues ella requeriría precisar qué es animal para luego indagar en lo racional, Descartes opta por continuar los pasos de sus consideraciones. Así es como el pensamiento, a diferencia del cuerpo, se muestra como aquello sin lo cual sería imposible determinar la existencia del Yo. No habría existencia sin un para alguien, al cual le es dada la existencia, ya que incluso los datos posiblemente falsos, como los del cuerpo, le vienen dados a ese alguien: al Yo pensante (1996:20-23).

${ }^{16}$ La mencionada diferencia cuantitativa entre cuerpo y cuerpo vivo mediante el modo de ser de la mera presencia espacial la podemos encontrar en Heidegger, quien, distinguiendo los fenómenos que aprehenden los conceptos Körper y Leib, afirma: "La diferencia de los límites del cuerpo (Körper) y cuerpo vivo (Leib) radicaría según esto en que los límites del cuerpo vivo (Leib) habrían seguido siendo empujados más allá que los límites del cuerpo (Körper) de tal manera que la diferencia de los límites sería cuantitativa” (1994:112).
} 
La última consideración que debemos hacer a partir de la idea de cuerpo en Descartes apunta a la realización misma de la experiencia de cuerpo que aquí subyace. En otras palabras, debemos atender a aquel tipo de experiencia mediante la cual el aparecer del cuerpo humano - donado al modo de lo extenso - es realizado, lo cual, en efecto, había sido una tarea que habíamos dejado hasta ahora pendiente. Hemos dicho que la comprensión moderna del cuerpo humano realza las partes por sobre el todo. También, que el concepto organismo implica esencialmente ser un compuesto, un ensamblaje de funciones ejecutadas por unidades menores: los órganos. Así, podemos apreciar que el tipo de experiencia que acá se despliega apunta a la división de la materia, a la fragmentación, en busca de un sustento material último. Se ha expuesto, también, que el ser del cuerpo - bajo esta interpretación- no es más que lo que él abarca espacialmente mediante su extensión. Todos estos caracteres nos hacen entender, en última instancia, que el acceso al fenómeno cuerpo humano no se despliega sino al modo de la cantidad, pues dicho modo de ser - a saber, lo cuantitativo - es aquel que privilegia la segmentación y la presencia del ente en cuanto volumen, al momento de acceder al mismo. Sólo si el ente es aprehendido mediante un modo de acceso cuantitativo será posible tratarlo fragmentariamente, pues cantidad significa, justamente, la posibilidad de división de la materia para delimitar la totalidad mediante la suma de las partes y las partes del organismo, en este sentido, no son sino partes de su composición material. ${ }^{17}$ Nos encontramos, pues, con una experiencia del cuerpo humano que no sólo lo despliega al interior de un modo de ser que pareciera serle inapropiado, sino, más aún, que le priva de aquello que precisamente pareciera caracterizarlo en su ser más propio: la vida. La experiencia cuantitativa de la corporalidad humana, podemos decirlo así, no es más que la desvivificación de un fenómeno que primariamente habría que aprehenderlo en su más propia vitalidad.

Pues bien, si anteriormente decíamos que el papel de Descartes se torna relevante en la presente discusión, habrá que entender que dicha relevancia no radica en que su tratamiento del cuerpo haya servido a modo de un fundamento teórico, el cual posteriormente habría sido aplicado por la tradición en el tratamiento concreto de todo cuerpo, incluyendo el humano, y que ahora bastaría sólo con reemplazarlo por fundamentos teóricos nuevos. El problema consiste, fundamentalmente, en que la interpretación mecanicista cartesiana parece ser la consolidación de un tipo específico de acceso al fenómeno

\footnotetext{
${ }^{17}$ El modo de acceso al ente que ahora es denominado cuántico atiende a la categoría de posón, tratada por Aristóteles como un modo de acceso, al interior del cual el ente dado se nos presenta con los atributos de la masa divisible. Cfr. Met. Libro D, Cap. 25; Libro Z, Cap. 10; Libro K, Cap. 3.
} 


\section{Felipe Johnson}

cuerpo humano, a saber, lo cuantitativo, que se ha desplegado en un tipo de experiencia fundamental de la corporalidad, desde la cual las modernas concepciones científicas e, incluso, en muchos casos también filosóficas, han seguido desarrollándose y esto a pesar de su distanciamiento explícito con Descartes y su crítica constante al cartesianismo. ${ }^{18}$ Lo decisivo es, en definitiva, destacar que la idea de cuerpo como "último soporte de toda función posible” es aquella idea esencial que subyace, incluso, en tematizaciones de la corporalidad que pretenden ganar una lucidez estricta acerca del modo de ser auténtico del cuerpo humano, caracterizando, no obstante, el fenómeno en cuestión mediante términos como "apoyo material”. ${ }^{19}$ En otras palabras, con Descartes se ha consolidado un acceso al fenómeno cuerpo humano y el ente que aparece mediante este acceso se ha vuelto el referente principal para las consideraciones sobre la corporalidad humana.

Sin embargo, habría que decir que la pregunta filosófica por el cuerpo no acaba con esta discusión; por el contrario, con ella recién comienza. Establecer que la corporalidad humana en su comprensión actual se despliega al interior de la idea de extensión, en el horizonte de lo cuantitativo, y que el estudio sobre su ser viene dado de la comprensión del ser en cuanto presencia espacial $^{20}$ significa sólo una advertencia metódica, un llamado a revisar nuevamente este acceso, para decidir si efectivamente corresponde al fenómeno que nos ocupa: ¿Es reductible el cuerpo humano a lo físico? ¿Son sus movimientos determinables al modo de meras funciones? ¿Son dichas funciones el resultado de un sustento material? Si bajo esta mirada son las partes las que parecen ganar primacía, ¿De dónde les viene dada su unidad esencial? ¿En qué radica fundamentalmente la vida del cuerpo humano?

\footnotetext{
${ }^{18}$ A modo de ejemplo podríamos, por un lado, mencionar a Plessner, en cuya obra principal esta experiencia de la corporalidad humana se refleja cuando él, en el prólogo a la segunda edición de su obra principal, afirma, criticando a Heidegger, que éste, en beneficio de su analítica existenciaria, habría descuidado las "condiciones físicas de la existencia" (die physische Bedingungen der Existenz) (1975:XIII). En la llamada fenomenología del cuerpo en Francia, por otro lado, hallamos también dicha experiencia cuando Alfonse de Waehlens en su prólogo a La structure du comportement de Merleau-Ponty afirma que la donación de lo real, tal como se expondría en Sein und Zeit, presupone que el sujeto cotidiano sea un ente corporal. En el citado prólogo podemos leer: "Ahora bien, los proyectos que, según Ser y Tiempo, engendran para nosotros la inteligibilidad de lo real, ya presuponen que el sujeto de la existencia cotidiana levante el brazo en cuanto él clava y forja” (V).

${ }^{19}$ Es el caso, por ejemplo, de Merleau-Ponty, quien, utilizando el concepto appui matériel, destacaría justamente la primacía del cuerpo vivo como condición de posibilidad de todo modo de instalarse en un mundo (moyen d'insertion) (1945:176) donde se realiza una analogía del cuerpo vivo con la obra de arte, pues, de acuerdo a sus maneras de ser, ninguno de los dos podrían carecer de la presencia física para ser tal.

${ }^{20}$ Utilizamos "presencia espacial” como la traducción estándar del modo de ser que Heidegger denominó Vorhandenheit, a diferencia de los llamados Zuhandenheit y Dasein.
} 
Hacia la pregunta por la corporalidad: reflexiones sobre el cuerpo humano

Estas preguntas parecieran exigir otro camino en vistas a su elucidación. Si la corporalidad humana es aquello que debe ser interrogado, el planteamiento de su pregunta deberá desarrollarse estrictamente al interior de lo humano, pues aquello es lo distintivo, la diferencia última de su modo de ser. Hemos visto que desde la comprensión de la corporalidad según el modo de ser de la mera presencia espacial, lo físico es aquel carácter que gana primacía. Lo que hemos interrogado, hasta ahora, ha sido sólo una cosa física que ejecuta funciones y a ella la hemos denominado "cuerpo vivo". Justamente, de esta comprensión deberá apartarse, en principio, todo análisis de la corporalidad humana, siendo así que es el carácter de lo físico - y todo lo que guarde relación con él sea todo aquello que se explicite en su segmentación en órganos o en su segmentación fisiológica - el primer carácter corporal que deberá ser puesto bajo tela de juicio. Es aquí donde la pregunta por lo humano muestra su primacía metódica en la problemática del cuerpo, pues con ella se anuncia una manera de ser específica a la luz de la cual la corporalidad se podría mostrar como lo que auténticamente es, a saber, como humana. En fin, el resultado último que quisiéramos destacar a partir de las discusiones anteriores es el hecho de que no sólo el tratamiento del cuerpo humano en sus caracteres propios debe seguir siendo precisado, sino que la pregunta misma por el fenómeno cuerpo humano exige aún una articulación estricta, de manera tal que aquello que mediante la pregunta sea convocado sea algo más que una cosa meramente extensa y sin vida.

\author{
Universidad Andres Bello* \\ Facultad de Humanidades y Educación \\ Licenciatura en Filosofía \\ Fernández Concha 700 / Las Condes, Santiago (Chile) \\ fjohnsonm@hotmail.com
}


Felipe Johnson

\section{BIBLIOGRAFÍA}

ARISTÓTELES. Metafísica. Londres: Oxford University Press, 1957.

BICHAT, Marie-François-Xavier. "Recherches physiologiques sur la vie et la mort”, en Oeuvres complètes. Vol. I. Paris: J. S. Chaudé Chaudé, Libraire-Éditeur, 1832.

DESCARTES, René. “La Description du Corps Humain”, en Oeuvres complètes. Vol. XI. (Ed.) Ch. Adam \& P. Tannery. Paris: Vrin, 1996.

"Les méditations metaphysiques", en Oeuvres complètes. Vol. IX. (Ed.). Ch. Adam \& P. Tannery. Paris: Vrin, 1996.

HEIDEGGER, Martin. Die Grundbegriffe der Metaphysik. Welt-EndlichkeitEinsamkeit. GA 29/30. Frankfurt a. M.: Vittorio Klostermann, 2004.

------- Sein und Zeit. Tübingen: Max Niemeyer Verlag, 2001.

------- Zollikoner Seminare. Frankfurt a. M.: Vittorio Klostermann, 1994.

------- Nietzsches Lehre vom Willen zur Macht als Erkenntnis. GA 47. Frankfurt a. M.: Vittorio Klostermann, 1989.

------ Nietzsche: Der europäische Nihilismus. GA 48. Frankfurt a. M.: Vittorio Klostermann, 1986.

HUSSERL, Edmund. Ideen zu einer reinen Phänomenologie und phänomenologischen Philosophie. Libro Segundo: Phänomenologis-chen Untersuchungen zur Konstitution. Husserliana. Vol. IV. Den Haag: Martinus Nijhoff Verlag, 1952.

JASPERS, Karl. "Die Idee des Arztes”, en Philosophie und Welt. Reden und Aufsätze. München: R. Piper \& Co. Verlag, 1958.

------- “Arzt und Patient”, en Philosophie und Welt. Reden und Aufsätze. München: R. Piper \& Co. Verlag, 1958.

MERLEAU-PONTY, Maurice. La structure du comportement. Paris: Quadrige/PUF, 1990.

------- Phénoménologie de la perception. Paris: Librairie Gallimard, 1945.

NIETZSCHE, Friedrich. “Nachlab 1885-1887”, en Kritische Studienausgabe. Vol. 12. (Ed.). G. Colli \& M. Montinari. Berlin, N. Y.: Walter de Gruyter, 1999.

PLESSNER, Helmuth. Die Stufen des Organischen und der Mensch. Berlin, N. Y.: Walter de Gruyter, 1975. 